\title{
Coefficient of restitution for rigid body dynamics modelling from onsite experimental data
}

\author{
F.R.P. Basson Newmont Asia Pacific, Australia \\ R. Humphreys Newmont Asia Pacific, Australia
}

A. Temmu Newmont Asia Pacific, Australia

\begin{abstract}
Rigid body dynamics uses the Newtonian laws of motion to solve the physics behaviour of moving bodies as a function of time. This approach is fast enough for real time simulation of multiple fall bodies, and can simulate the trajectories of three-dimensional volumetric bodies during free fall, bouncing, sliding and rolling. An additional benefit of rigid body dynamics is that the input parameters required are few, measurable and intuitive, as only the coefficient of restitution (COR), and the static and dynamic friction angles are required.
\end{abstract}

The Geotechnical Team at Newmont Boddington Gold (Boddington) undertook simple experiments to determine the ranges of COR values applicable to different surfaces for the Boddington pits. Ten rocks between 0.35 and $2.08 \mathrm{~kg}$ were collected, weighed, marked, and measured. Each rock was then dropped ten times from a height of $1.40 \mathrm{~m}$ onto the four different horizontal pit surfaces 'pit floor', 'haul road', 'catch berm', and 'hard rock'. The rebound of each drop test was measured with a scaled white board behind the test area from video footage. A total of 400 data points were collected and the results analysed. It was found that the different rebound surfaces have different levels of predictability in rebound behaviour, and that some surfaces a more prone to occasional outlier results. The aim of these experiments was to obtain rebound information for use in the rigid body dynamics modelling software package Trajec3D.

During a full scale rockfall experiment, and the accompanying three-dimensional back-analysis with volumetric fall bodies, many factors determine the outcome that complicates the verification of the individual simulation components. The simple experiments discussed before were simulated in Trajec3D to determine if rigid body dynamics could simulate realistic rebound behaviour with different rock shapes, sizes, and physics material interaction properties.

The combined outcomes from the rockfall experiments and rigid body dynamic simulations lead to the identification of the critical factors controlling fall body rebound behaviour at Boddington. Appropriate COR values will be selected and used in the analysis of larger scale rockfall experiments, the back-analyses of known rockfall events, and to identify and assess potential future rockfall areas.

\section{Introduction}

Trajec3D (Basson, 2013) is a three-dimensional, rigid body dynamics, rockfall software package that can simulate the trajectory of volumetric bodies during free fall, bouncing, sliding and rolling. The physics interaction between materials requires only three measurable and intuitive input parameters between the materials: the coefficient of restitution, the static friction angle, and the dynamic friction angle (Basson, 2012).

The coefficient of restitution (COR) defines the elasticity or bounciness of the interaction, and represents the ratio of the speeds after and before impact taken along the line of impact. A COR value of one indicates a perfectly elastic collision with no loss in velocity and thus no loss in energy. A value of zero implies a perfectly plastic collision where all the velocity along the line of impact is absorbed. 
The COR is defined as:

$$
C_{R}=\frac{v}{V}
$$

Where:

$$
\begin{aligned}
& C_{R}=\text { coefficient of restitution. } \\
& v=\text { scalar velocity of the fall body after impact. } \\
& V=\text { scalar velocity of the fall body before impact. }
\end{aligned}
$$

When the fall object starts from a zero velocity and bounces from a stationary object, the formula can also be written as (Basson, 2012):

$$
C_{R}=\sqrt{\frac{h}{H}}
$$

Where:

$$
\begin{aligned}
& h=\text { bounce height } . \\
& H=\text { drop height. }
\end{aligned}
$$

During a full scale rockfall back-analysis, many factors determine the outcome which complicates the verification of the individual simulation components. Controlled experiments were thus undertaken by the Geotechnical Team at Newmont Boddington Gold (Boddington) to determine the ranges of COR values applicable to different rock surfaces for the Boddington pits. These simple experiments were then simulated in Trajec3D for different fall body shapes and sizes to determine if rigid body dynamics could simulate realistic rebound behaviour.

\section{Site experiments}

\subsection{Site experiment set-up}

Ten random rocks were collected, marked, weighed, and photographed with a ruler to estimate the dimensions of each rock.

\begin{tabular}{|c|c|c|c|c|}
\hline Rock 1: $0.85 \mathrm{~kg}$ & Rock 2: $0.56 \mathrm{~kg}$ & Rock 3: $1.46 \mathrm{~kg}$ & Rock 4: $0.35 \mathrm{~kg}$ & Rock 5: $0.73 \mathrm{~kg}$ \\
\hline & & & & \\
\hline & & & & \\
\hline & & & & \\
\hline
\end{tabular}

Figure 1 Rocks selected as fall bodies

Four typical floor surfaces around the pit were selected, comprised of the 'pit floor', 'haul road', 'catch berm' and 'hard rock' surfaces as shown in Figure 2. 


\begin{tabular}{|c|c|c|c|}
\hline (a) Pit Floor & (b) Haul Road & (c) Catch Berm & (d) Hard Rock \\
\hline & & & \\
\hline & & & \\
& & & \\
\hline
\end{tabular}

\section{Figure 2 Rock surfaces selected for testing}

The rocks were manually lifted to $1.40 \mathrm{~m}$ from the floor and dropped. Each rebound was measured by comparing the rebound distance with the scaled white board that was placed behind the drop test area. Due to the speed of the rocks, the Geotechnical Team filmed each test and gathered the rebound information by replaying the videos in slow motion. Ten drops were undertaken for each rock on each of the four surfaces, giving a total of 400 data points. As small rebounds are impossible to estimate, the minimum rebound was capped to $1 \mathrm{~mm}$, thus all rocks were assumed to have at least $1 \mathrm{~mm}$ of rebound from the rock surface which equates to a COR value of 0.003 .

\subsection{Results from the site experiment}

Many correlations and distributions were gathered from this experiment that can be used for future Boddington site specific analyses.

\subsubsection{COR histograms by rebound surface}

Rebound results are displayed in Figure 3 as COR frequency histograms for all rock surfaces.

The most common (mode) COR values for the different surfaces are: 0.003 for both the 'catch berm' and 'pit floor', 0.085 for the 'haul road', and 0.120 for the 'hard rock'. The 'catch berm' was the most predictable rebound surface with the highest number of very low rebounds and the least scatter in the results.

The maximum COR value outlier for the 'hard rock' surface is not an error reading. Out of the 400 rocks that were dropped, one behaved differently to the others for no apparent reason. 'Rock 3' is $1.46 \mathrm{~kg}$, and when dropped from a height of $1.40 \mathrm{~m}$ rebounded $0.57 \mathrm{~m}$ for a COR value of 0.64 . The closest other results to this outcome were three rocks with COR values of 0.50 , rebounding $0.35 \mathrm{~m}$ after being dropped from a $1.40 \mathrm{~m}$ height onto the 'hard rock' surface. This is only $60 \%$ of the height of the outlier bounce. 


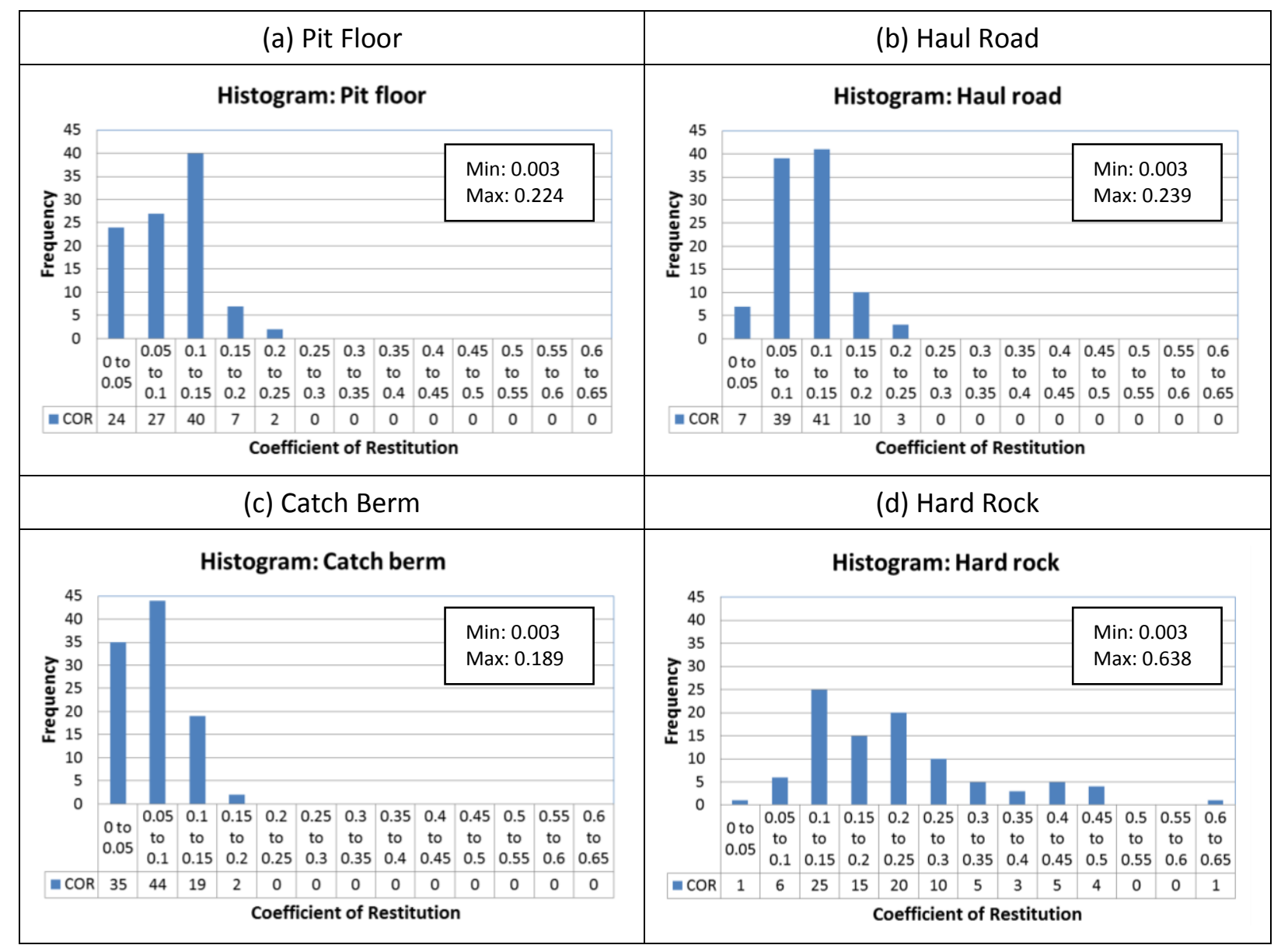

Figure 3 COR histograms for all rock surfaces

\subsubsection{Median COR values by rock weight}

The median COR value was determined for each rock on each surface, and the median value is plotted in Figure 4 against the weight of each rock.

The 'pit floor' rebounds seem unaffected by the rock weight, but the median COR value of all other surfaces decreases with weight. This is an expected outcome, as larger rockfalls will penetrate surfaces covered with small rock fragments deeper, have a longer impact time, and increased energy absorption. 


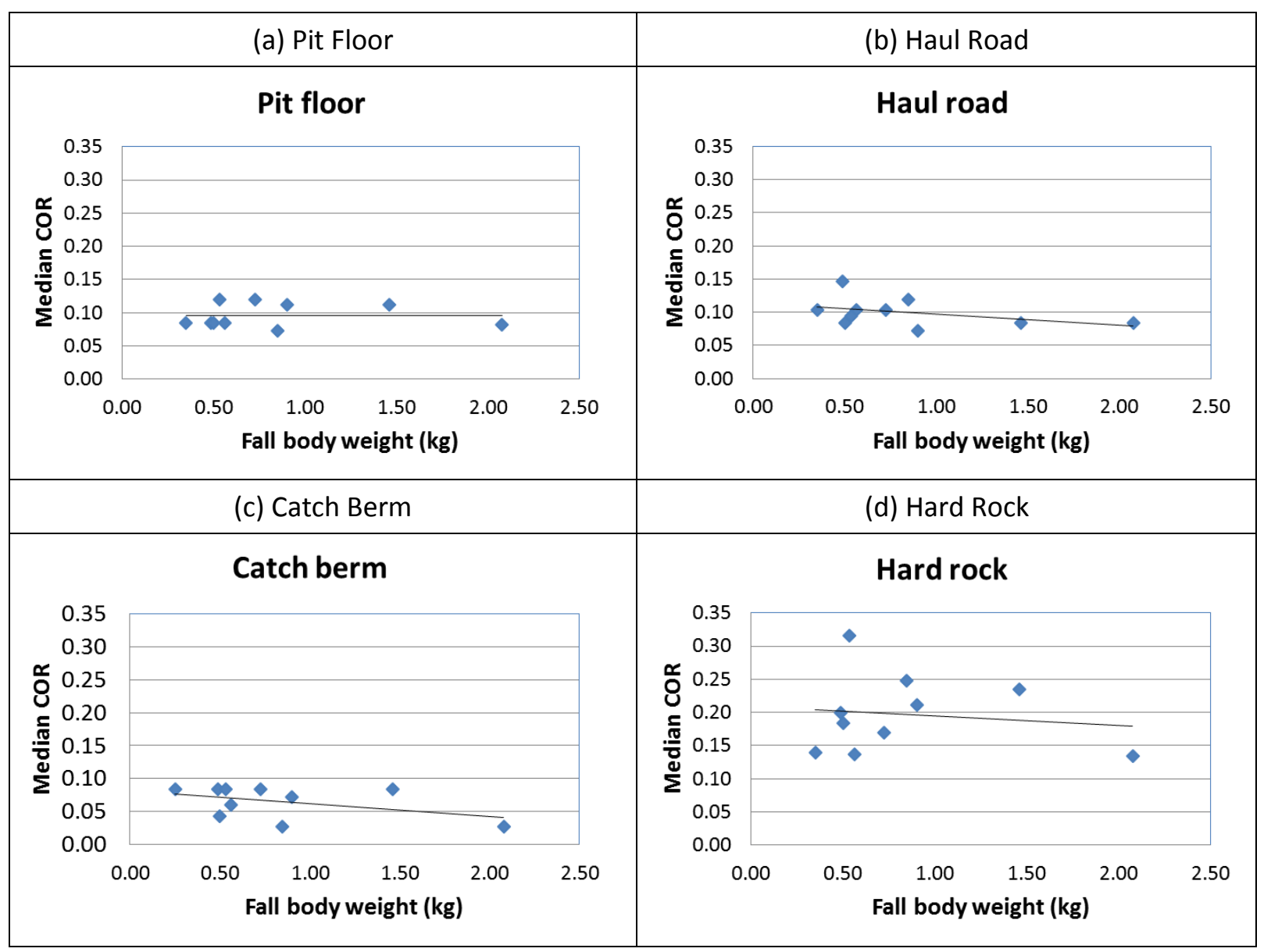

Figure 4 Median COR values against the fall body weight

\section{Trajec3D experiments}

The next step was to confirm if the Newton Physics Engine (Jerez and Suero, 2012) used in Trajec3D could consistently reproduce the selected COR values during simulations. A study was done by the University of Western Australia (Boeing, 2007) where different physics engines were compared against five tests; integrator performance, accuracy of material properties, constraint stability, collision system, and stacking test. Of most importance for the study in this paper was the material properties test, as materials are responsible for simulating friction and rebound properties during collisions.

Boeing (2007) concluded that no single engine performed best at all tasks, as almost every test was performed best by a different engine. The Newton Physics Engine was the best performing engine with friction tests, but scored reasonably poorly in their restitution test. As the friction angle outcome agrees with the authors' experience, this will not be further investigated in this paper. The poor restitution outcome was not in agreement with the authors' experience and experimental tests were constructed. The results are discussed in the following sections.

\subsection{Trajec3D experiment setup}

Control experiments were constructed in Trajec3D that are similar to the site experiments discussed in the previous section. Two set-ups were selected; the first to investigate the rebound of perfect spheres, and the second to determine the impact of different fall body shapes. For all cases illustrated, a high COR value of 0.50 was used to provide visually pronounced fall body rebounds. 


\subsubsection{Coefficient of restitution of mathematical spheres}

A plane was constructed above the surface at a slight angle, and perfect spheres dropped from the plane that acted as a collision strip to generate the fall bodies. Mathematical (perfect) spheres were selected to eliminate the impact of the fall body shape. A vertical strip will produce slightly more precise results, but the rebounds then occur in the same line as the initial fall paths, and the rebound peaks are difficult to pick. Tests were run with different collision strip angles, and the results were only marginally impacted if the angle is $10^{\circ}$ or less from vertical.

Equation 2 (Section 1 ) can be used to calculate the COR value of the rebound as the drop height $(H)$ is known and the first peak represents the rebound height $(h)$. The COR value of consecutive bounces can also be determined by using the earlier rebound peak as the drop height, and the following peak as the rebound height. These calculations can then be used to determine if the Newton Physics Engine accurately solves for the rebound from the selected material properties.

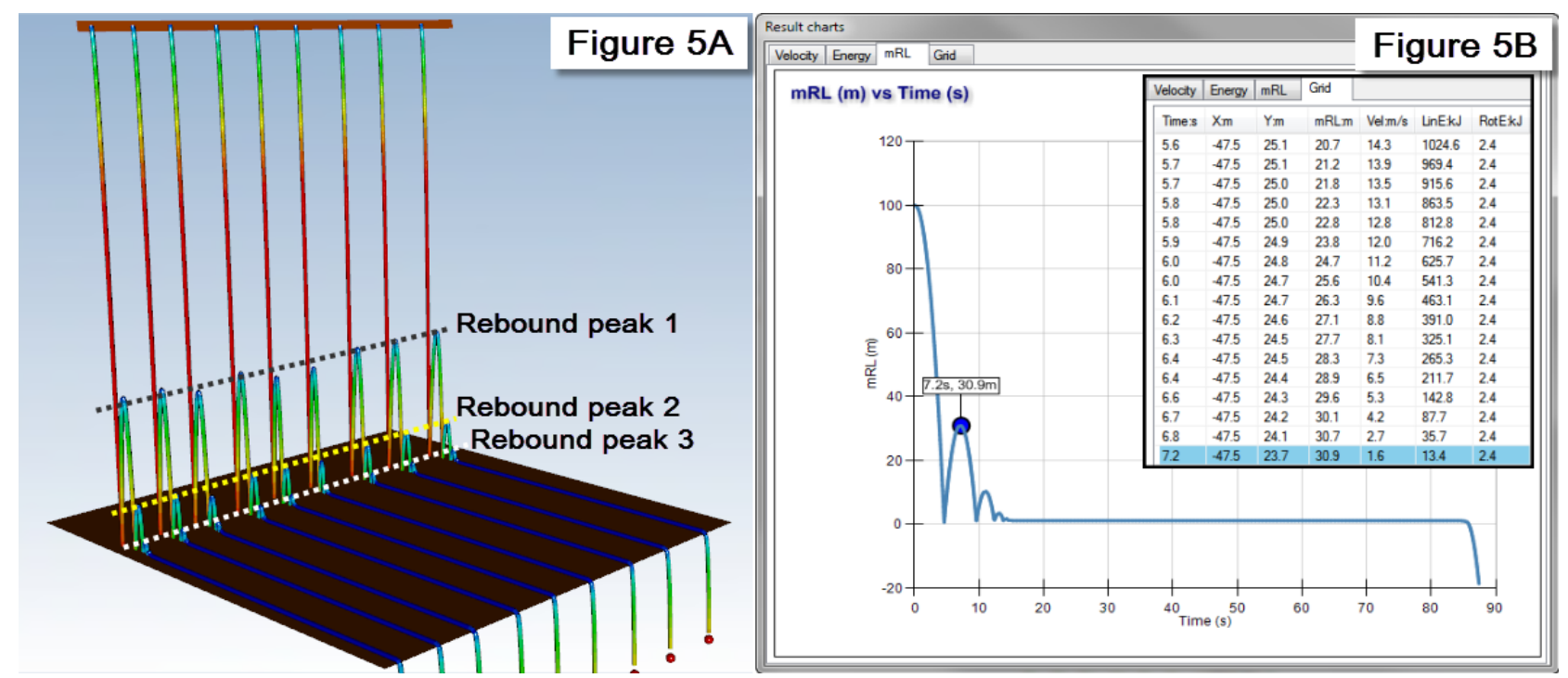

Figure 5 Simulated rebounds for perfect spheres $(C O R=0.50)$

Figure 5(a) shows the setup used and the variability of the rebounds calculated by Trajec3D is obvious from the different rebound peak heights. One of the graphs in Trajec3D is called ' $\mathrm{mRL}$ ' and provides the vertical displacement for selected fall body paths. The exact values can also be obtained for each fall body path from the 'Grid' option shown as insert to Figure 5(b).

\subsubsection{Rebound of different fall body shapes}

In this experiment, the same slightly dipping plane was again used as collision strip. This plane results in a slight tilt of the fall body to ensure the symmetrical fall bodies rebound from their edges. Figures 6(a) and 6(b) show example outcomes from this experiment. Figure 6(c) is an energy distribution graph differentiating between translational and rotational energies, and Figure $6(\mathrm{~d})$ shows the fall body shapes used. 


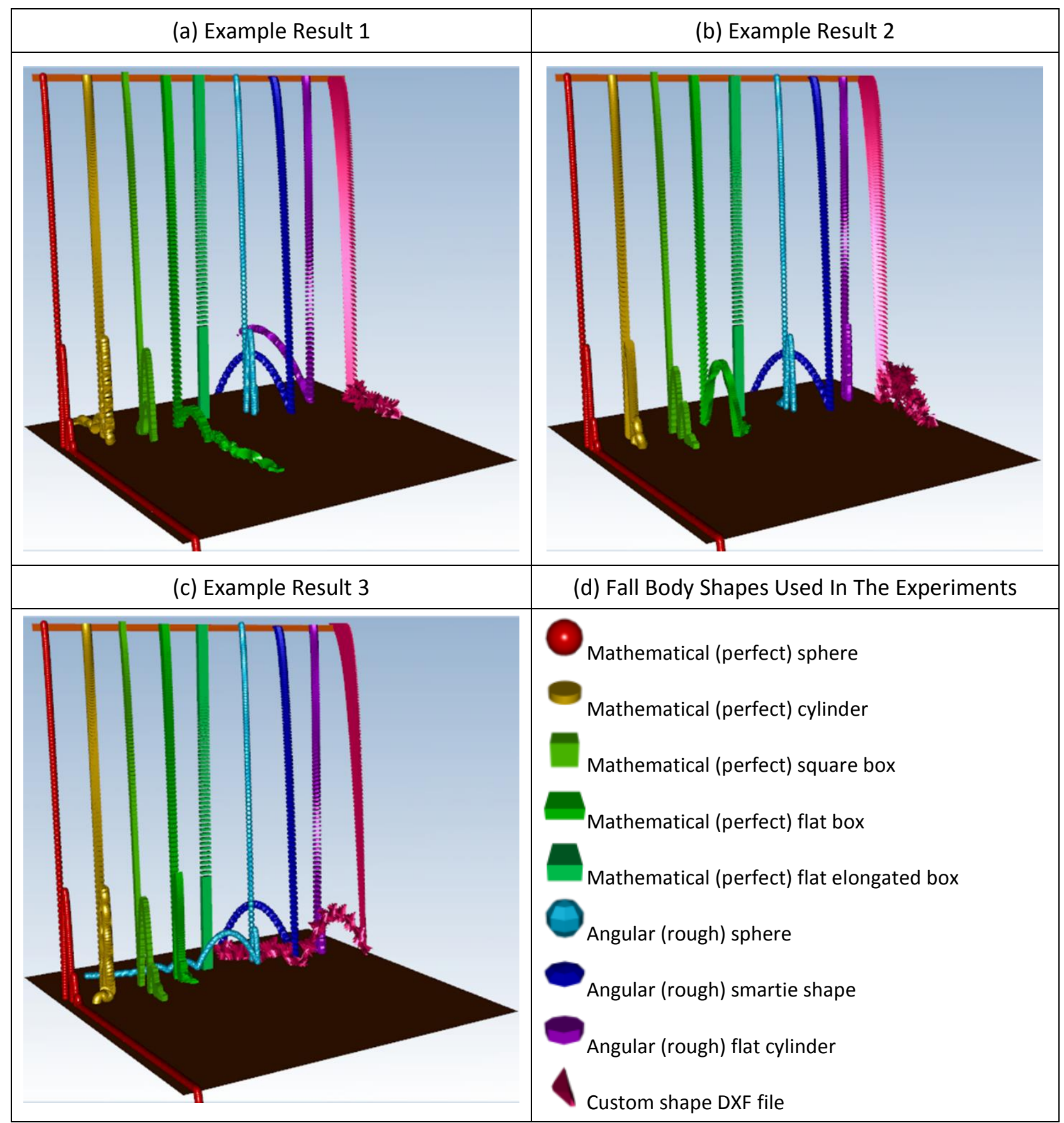

Figure 6 Simulated rebounds from different shapes $(C O R=0.5)$

\subsection{Results from the Trajec3D experiments}

\subsubsection{Coefficient of restitution of mathematical spheres}

Different sphere sizes and drop heights were used, but the results were not markedly affected by these changes. Figure 7 shows the COR values for three consecutive bounces of a single fall body with a physics material interaction with a COR value of 0.50. Many more tests were done, but the results below are representative of all outcomes. 


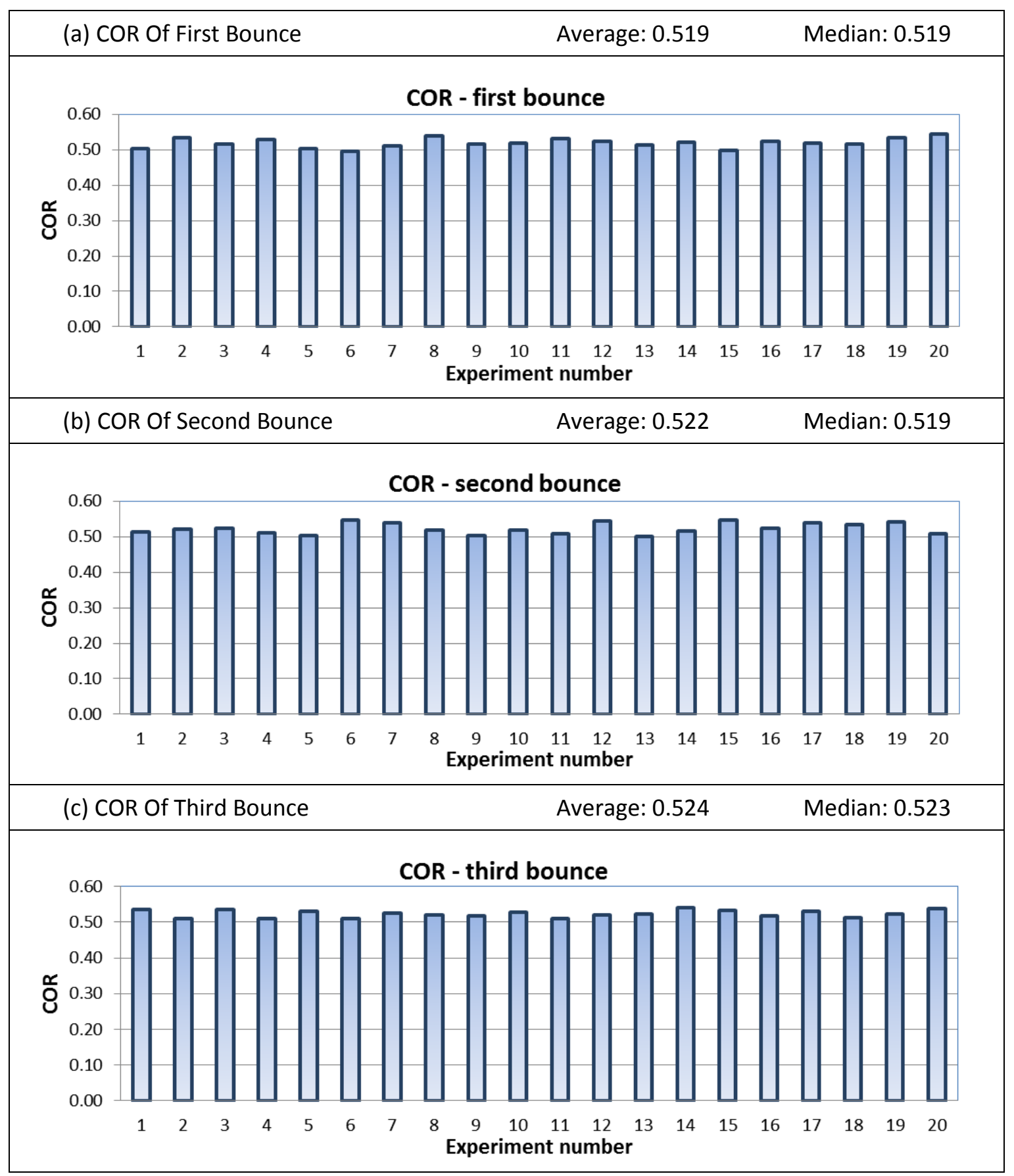

Figure 7 Rebound outcomes from the Newton Physics Engine for a COR $=0.50$

The Newton Physics Engine is reasonably accurate in reproducing the COR value selected for the physics material interaction. In all cases the rebounds were either the mathematical rebound value, or slightly higher. Typically the Newton Physics Engine calculates rebounds with COR values of 0.02 higher than the selected material interaction value.

\subsubsection{Rebound of different fall body shapes}

Fall body shape has a huge impact on rebound behaviour. Near-spherical shapes have a predictable rebound path, but angular and elongated shapes behave more unpredictably. Angular and elongated shapes often bounce at an angle to the initial movement, and they could even bounce in the opposite direction to the initial velocity, as shown in Figure 6. 
Investigating the energy graphs of the fall body paths shows that the rotational energy (see Figure 6(c)) plays a more pronounced role after rebound with angular and elongated shapes compared to spherical fall bodies. These rotational energies then further contribute to the occasional erratic bounce behaviour of these fall bodies. Rocscience Inc. (Vijayakumar et al., 2012) showed that measured COR values can range from negative to larger than 1.00 due to eccentricity of the rock shape and its rotational energy. This seems to violate the law of conservation of energy, but is only a function of the way the COR is defined, whereby the transformations between translational and rotational energies are unaccounted for.

\section{$4 \quad$ Summary}

\subsection{Site experiments}

Hard rock surfaces resulted in higher rebounds, more variable rebound behaviour, and occasionally, excessive outlier rebounds. Soft rock surfaces such as catch berms resulted in more controlled and predictable rebound behaviour. All surfaces had instances where the rebounds were too small to be observed, and these were most common for the 'catch berm' surface. A COR value of 0.10 seems to be an upper limit for all surfaces except the hard rock surface, but the COR value generally decreases with fall body weight.

The least rebound and most predictable behaviour were recorded for the 'catch berm', followed by the 'pit floor' and 'haul road'. Rebound on the hard rock surface was higher than on any other surface.

\subsection{Trajec3D experiments}

The University of Western Australia (Boeing, 2007) tested many physics engine, and the Newton Physics Engine performed best at the friction tests. COR tests by the author showed that the Newton Physics Engine is also reasonably accurate in reproducing the COR value selected for the physics material interaction. Rebounds either agreed with the calculated analytical solution or were slightly higher, with a typical positive COR error of +0.02 .

Simulations with the Newton Physics Engine reproduced actual fall body behaviour, whereby near-spherical shapes have predictable rebound paths, but angular and elongated shapes behave less predictable. The rotational energy also typically plays a more pronounced role after rebound with angular and elongated shapes than with spherical fall bodies, contributing to the less predictable nature of their bounces.

\section{Conclusions}

The potential for rebounds from hard surfaces should be minimised especially when rocks could fall from substantial heights. The first rebound after free falling from a substantial height will largely determine the impact area of the fall body. Angular and elongated fall bodies could bounce in unexpected directions, and have a higher probability for large COR values after the first bounce, due to the potential of a rotational energy component.

The Trajec3D experiments confirmed that the Newton Physics Engine reproduces actual physics behaviour of fall bodies sufficiently accurately to use as a rockfall simulation tool to assess potential outcomes in real life situations. As with all modelling results, the outcomes have to be intelligently evaluated, and engineering judgement is required to assess the value of a particular simulation result.

Numerical modelling software packages are educational tools, and in the case of Trajec3D, the potential benefits are (Basson, 2012):

- Visualise potential rockfall patterns.

- Qualify potential outcomes - for example one in five rocks from a specific bench face will end up on a ramp.

- Comparative and sensitivity studies with different variables. 
- Exposing surprise events overlooked by visual inspection.

- Educational tool (toy) that opens the imagination to potential outcomes.

\section{$6 \quad$ Further work}

The next step is to back-analyse actual rockfall events, and determine suitable parameters that will produce realistic outcomes of complex rock trajectories that could include free fall, bouncing, sliding and rolling.

\section{Acknowledgement}

The authors gratefully acknowledge the permission given by Newmont Asia Pacific, and Newmont Boddington Gold in particular, to publish this paper. Support from the rest of the site geotechnical team Tim Rees, Tata Peryoga, Gavin McCartney, and Nathan Dalton is highly appreciated.

Also for Marc Elmouttie that provided the authors with a custom shape fall body in DXF format generated with the fracture network software developed for the Large Open Pit Mine Slope Stability Project.

\section{References}

Basson, F.R.P. (2012) Rigid body dynamics for rock fall trajectory simulation, in Proceedings 46th US Rock Mechanics/Geomechanics Symposium 2012, A. Bobet, R. Ewy, M. Gadde, J. Labuz, L. Pyrak-Nolte, A. Tutuncu and E. Westman (eds), $24-27$ June, Chicago, USA, Curran Associates Inc, New York, pp. 1438-1444.

Basson, F.R.P. (2013) BasRock Software for Geotechs, Perth, viewed 20 April 2013, http://www.basrock.com/page13.htm.

Boeing, A. (2007) Evaluation of real-time physics simulation systems, in Proceedings 5th International Conference on Computer Graphics and Interactive Techniques in Australasia and South East Asia (GRAPHITE 2007 Conference), 1-4 December 2007, Perth, Australia, ACM, New York.

Jerez, J. and Suero, A. (2012) Newton Game Dynamics, viewed 20 April 2013, http://newtondynamics.com/forum/newton.php.

Vijayakumar, S., Yacoub, T., Ranjram, M. and Curran, J.H. (2012) Effect of Rockfall Shape on Normal Coefficient of Restitution, in Proceedings 46th US Rock Mechanics Symposium 2012, A. Bobet, R. Ewy, M. Gadde, J. Labuz, L. Pyrak-Nolte, A. Tutuncu, and E. Westman (eds), 24-27 June, 2012, Chicago, USA, Curran Associates Inc, New York, pp. 1420-1427. 